\title{
A CHARACTERIZATION OF STEINITZ GROUP RINGS
}

\author{
PAUL J. ALLEN AND JOSEPH NEGGERS
}

ABSTRACT. A ring $R$ with an identity is a (right) Steinitz ring provided any linearly independent subset of a free (right) $R$-module can be extended to a basis for the module by adjoining elements from any given basis. In this paper, we characterize those group rings which are Steinitz rings by the following:

Theorem. The group ring $R[G]$ is a Steinitz ring if and only if $R$ is a Steinitz ring and either (1) char $R=p^{i}$ and $G$ is a finite p-group or (2) char $R=0$ and $G=1$.

Introduction. A ring $R$ with an identity will be called a (right) Steinitz ring provided any linearly independent subset of a free (right) $R$-module can be extended to a basis for the module by adjoining elements from any given basis. A subset $S$ of the ring $R$ will be called $T$-nilpotent if for each sequence $\left\{x_{i}\right\}_{i=1}^{\infty}$ in $S$, there exists an integer $n$ such that $x_{n} x_{n-1} \cdots x_{1}=0$. Chwe and Neggers [1], [2] proved that $R$ is a Steinitz ring if and only if $R$ is a local ring (i.e., the Jacobson radical is the set of nonunits) with a $T$-nilpotent Jacobson radical. In this paper we characterize those group rings which are Steinitz rings.

A characterization of Steinitz group rings. Since Steinitz rings have characteristic 0 or $p^{i}$ where $p$ is a prime, our characterization consists of two cases and is stated as follows:

Theorem. The group ring $R[G]$ is a Steinitz ring if and only if $R$ is a Steinitz ring and either (1) char $R=p^{i}$ and $G$ is a finite p-group or (2) char $R=0$ and $G=1$.

Proof. Suppose that $R[G]$ is a Steinitz group ring. Since the map $\nu$ defined by $\left(\Sigma r_{g} g\right) \nu=\Sigma r_{g}$ is a homomorphism from $R[G]$ onto $R$, it follows that $R$ is a Steinitz ring. Since $(1-g) \nu=0$, it is clear that $1-g$ is a nonunit for every $g \in G$. Consequently, $\{1-g \mid g \in G\} \subseteq J(R[G])$ where $J(R[G])$ denotes the radical of $R[G]$. When $\alpha=\Sigma r_{g} g$ is an element of $R[G]$, the support of $\alpha$ will mean $\left\{\left.g \in G\right|_{g} \neq 0\right.$ in the representation $\left.\alpha=\Sigma r_{g} g\right\}$ and $r_{1}$ will

Received by the editors March 28, 1974.

AMS (MOS) subject classifications (1970). Primary 16A26.

Key words and phrases. Group ring, Steinitz ring, $T$-nilpotent Jacobson radical. 
be called the trace of $\alpha$. When $g_{1}, g_{2}, \cdots, g_{j-1}$ are elements of $G$, let $S_{j}$ denote the support of $\left(1-g_{j-1}\right)\left(1-g_{j-2}\right) \cdots\left(1-g_{1}\right)$. Suppose $g_{1}, g_{2}, \cdots$, $g_{n-1}$ have been chosen from $G$ such that the trace of $\left(1-g_{n-1}\right)\left(1-g_{n-2}\right)$ $\cdots\left(1-g_{1}\right)$ is 1. If $G_{n}=G-\left(S_{n} \cup S_{n}^{-1}\right) \neq \varnothing$, then for any $g_{n} \in G_{n}$, it follows that $\left(1-g_{n}\right)\left(1-g_{n-1}\right) \cdots\left(1-g_{1}\right)$ has trace 1 , hence the product is nonzero. Since $J(R[G])$ is $T$-nilpotent, $G_{n}=\varnothing$ for some $n$ and it follows that $G$ is a finite group.

The radical of a Steinitz ring contains all of the nonunits, thus for each element $g$, either $g$ or $1-g$ is a unit. It follows that the only idempotents in a Steinitz ring are 0 and 1. On the other hand, if $g$ is an element of order $n$ in the group $G$ and if $n$ is a unit in $R[G]$, then $n^{-1}\left(1+g+\cdots+g^{n-1}\right)$ is idempotent, hence $n^{-1}\left(1+g+\cdots+g^{n-1}\right)$ must be 0 or 1 . When char $R=0$, $n$ is always a unit and thus the finite group $G$ must be trivial. When char $R=$ $p^{i}$, it must follow for each $g \in G$ that $n=1$ or $p \mid n$. In this case, $G$ is a $p$ group.

If $R$ is a Steinitz ring and condition 2 holds, it is obvious that $R[G]$ is a Steinitz ring. When $R$ is a Steinitz ring and condition 1 holds, we will show $R[G]$ is a Steinitz ring with the aid of the following:

Lemma. Let $R$ be a ring of characteristic $p^{i}$ and let $1=Z_{0} \subset Z_{1} \subset \cdots \subset$ $Z_{m}=G$ be the ascending central series of the finite p-group $G$. For each $r=0,1, \cdots, m$ there exists an integer $n_{r}$ such that any sequence in $\{1-g \mid g \in G\}$ with at least $n_{r}$ terms of the form $1-z$ with $z \in Z_{r}$ has product zero in $R[G]$.

Proof. For any product $s$ of factors $1-x_{i}, x_{i} \in G$, we define $\gamma_{t}(s)$ to be the number of factors where $x_{i} \in Z_{t}$. When $\gamma_{0}(s) \geq 1$, it is clear that $s=0$. We may take $n_{0}=1$. Suppose $n_{r}$ is a number such that $\gamma_{r}(s) \geq n_{r}$ implies $s=0$. Let $n_{r+1}=n_{r}+n_{r}\left|Z_{r+1}\right| i p^{i}|G|$. We will show $\gamma_{r+1}(s) \geq n_{r+1}$ implies $s=0$. The Lemma will follow by induction because of the nilpotence of $G$.

We shall use a second induction step. Namely, it will be shown that if $0 \leq k \leq n_{r}$ and $s$ is a product with $\gamma_{r}(s) \geq k$ and $\gamma_{r+1}(s) \geq n_{r+1}-k$, then $s$ is a sum of products $s_{i}$ for which $\gamma_{r}\left(s_{i}\right) \geq k+1$ and $\gamma_{r+1}\left(s_{i}\right) \geq n_{r+1}-(k+1)$. Repeating this step at most $n_{r}$ times will show that if $\gamma_{r+1}(s) \geq n_{r+1}$, then $s$ is a sum of products $s_{i}$ for which $\gamma_{r}\left(s_{i}\right) \geq n_{r}$, and consequently $s=0$.

Suppose that $\gamma_{r}(s) \geq k$ and $\gamma_{r+1}(s) \geq n_{r+1}-k$, where $0 \leq k \leq n_{r}$. It follows from a pigeonhole argument that $s$ contains at least $n_{r} i p^{i}|G|$ equal factors $1-x$ for some $x \in Z_{r+1}$, since 


$$
\frac{n_{r+1}-k}{\left|Z_{r+1}\right|} \geq \frac{n_{r+1}-n_{r}}{\left|Z_{r+1}\right|}=n_{r} i p^{i}|G| \text {. }
$$

There is nothing to prove if $s=0$, so we may suppose that $\gamma_{r}(s)<n_{r}$. Therefore, there must be a product $\pi$ of consecutive factors in $s$ which contains $n=i p^{i}|G|$ of the equal factors $1-x$ and does not contain any factor $1-y$ for $y \in Z_{r}$. Restricting our attention to this subproduct $\pi$, we reorder the factors of $\pi$ to collect the equal factors $1-x$, using the relation

$$
(1-u)(1-x)=(1-x)(1-u)+u x\left(1-x^{-1} u^{-1} x u\right) \text {. }
$$

Since $(1-x)^{n}=0$, we know $s$ is zero plus a sum of products $s_{i}$ where each $s_{i}$ is obtained by replacing a product $(1-u)(1-x)$ in $\pi$ by $u x\left(1-x^{-1} u^{-1} x u\right)$. Because of our choice of $\pi$, we know $u \notin Z_{r}$ and $x \notin Z_{r}$. Since $x \in Z_{r+1}$, it follows that $x^{-1} u^{-1} x u \in Z_{r}$ and therefore $\gamma_{r}\left(s_{i}\right)=\gamma_{r}(s)+1 \geq k+1$. Since we have removed two factors from $s$ and introduced the new element $1-x^{-1} u^{-1} x u$, we have $\gamma_{r+1}\left(s_{i}\right) \geq \gamma_{r+1}(s)-1 \geq n_{r+1}-(k+1)$. The product $s_{i}$ contains the factor $u x$, but this is of no consequence since it can be moved to the far right of $s_{i}$ by using the relation $g(1-y)=\left(1-g y g^{-1}\right) g$. This does not change the numbers $\gamma_{t}$ since $Z_{t}$ is normal in $G$, and the proof of the Lemma is complete.

We are now ready to proceed with the proof of the Theorem. Suppose $R$ is a Steinitz ring of characteristic $p^{i}$ and $G$ is a finite $p$-group. When $S=$ $\{1-g \mid g \in G\}$, the Lemma implies there exists a positive integer $k=n_{m}$ such that $S^{k}=0$. Let

$$
N=\sum_{g \in G} R(1-g) .
$$

Since $x(1-g)=(1-x g)-(1-x)$, it is an easy matter to show $N$ is an ideal in the ring $R[G]$. Let $\left\{x_{1}, \cdots, x_{k}\right\}$ be any set of $k$ elements of $N$ where $x_{i}=$ $\Sigma_{g \in G}{ }^{r} i g(1-g)$. Since elements of $R$ commute with elements of $S, x_{k} x_{k-1}$ $\cdots x_{1}$ is clearly a sum of terms of the form

$$
r\left(1-g_{1}\right)\left(1-g_{2}\right) \cdots\left(1-g_{k}\right)
$$

and hence $N^{k}=0$.

Let $J(R)$ denote the radical of the Steinitz ring $R$. We know $J(R)$ is a $T$-nilpotent subset of $R$ consisting precisely of the nonunits of $R$. In general, the sum of a $T$-nilpotent subring and a $T$-nilpotent ideal is $T$-nilpotent, and in our setting we argue as follows: Since $J(R)+N / N$ is a homomorphic image of $J(R)$, we know $J(R)+N / N$ is a $T$-nilpotent set. Let $\left\{x_{i}\right\}_{i=1}^{\infty}$ be a 
sequence in $J(R)+N$. From the sequence $\left\{x_{i}+N\right\}$ in $J(R)+N / N$, we can choose integers $m_{0}=0, m_{1}, \cdots, m_{k}$ where $y_{j}=\Pi_{i=m_{j-1}+1}^{m_{j}} x_{i} \in N$ for $j=1$, $2, \cdots, k$. Clearly,

$$
x_{n_{k}} x_{n_{k-1}} \cdots x_{1}=y_{k} y_{k-1} \cdots y_{1}=0,
$$

since $N^{k}=0$ and it follows that $J(R)+N$ is a $T$-nilpotent subset of $R[G]$. Using the fact that $R[G]=R+N$, it is an easy matter to show $J(R)+N$ is an ideal in $R[G]$. Let $x \in R[G]$ where $x \notin J(R)+N$. Writing $x=u(1-z)$, where $u$ is a unit in $R$ and $z \in N$, one concludes immediately that

$$
x^{-1}=\left(1+z+\cdots+z^{k-1}\right) u^{-1}
$$

since $N^{k}=0$. Therefore, if $x \notin J(R)+N$, then $x$ is a unit in $R[G]$ and it follows that $J(R)+N$ is the ideal of nonunits in $R[G]$. It has now been shown that $R[G]$ is a Steinitz ring of characteristic $p^{i}$ when $R$ is a Steinitz ring of characteristic $p^{i}$ and $G$ is a finite $p$-group.

Note. Since Steinitz rings are perfect rings, one can use a result of S. M. Woods [4] to prove that $G$ is a finite group when $R[G]$ is a Steinitz ring. The direct proof given above depends on $R[G]$ being Steinitz rather than merely perfect. I. G. Connell (see [3, Theorem 9]) proved the fundamental (augmentation) ideal $N$ is nilpotent when $G$ is a finite $p$-group and $p$ is nilpotent in $R$. Connell's proof of this result is by induction on the order of $G$ and the proof only guarantees the existence of $k$ such that $N^{k}=0$. On the other hand, the proof of our Lemma gives a method by which a specific $k$ can be calculated when needed. Consequently, we presented our Lemma as an alternative rather than quoting Connell's result. In addition, we pose the following:

Problem. When $I=Z_{0} \subset Z_{1} \subset \cdots \subset Z_{m}=G$ is the ascending central series of the finite $p$-group $G$ and $R$ is a ring of characteristic $p^{i}$, find a better bound, or the best bound, for the smallest integer $k$ such that $N^{k}=0$.

\section{REFERENCES}

1. Byoung-song Chwe and Joseph Neggers, On the extension of linearly independent subsets of free modules to bases, Proc. Amer. Math. Soc. 24 (1970), 466470. MR $40 \# 5652$.

2. - Local rings with left vanishing radical, J. London Math. Soc. (2) 4 (1971), 374-378. MR $44 \# 6746$.

3. I. G. Connell, On the group ring, Canad. J. Math. 15 (1963), 650-685. MR $27 \# 3666$.

4. S. M. Woods, On perfect group rings, Proc. Amer. Math. Soc. 27 (1971), 4952. MR $42 \# 6130$. 